\title{
Specific proteolysis of the A-kinase-anchoring protein 149 at the Asp582 residue by caspases during apoptosis
}

\author{
HOESOOK YOO ${ }^{1 *}$, HWA JUN CHA ${ }^{1 *}$, JOONYOUNG LEE ${ }^{1}$, EUN-OK YU ${ }^{1}$, SEUNGHEE BAE ${ }^{1}$, \\ JIN HYUK JUNG ${ }^{1}$, INSOOK SOHN ${ }^{2}$, SU-JAE LEE ${ }^{3}$, KWANG-HEE YANG ${ }^{4}$, SANG-HYEOK WOO ${ }^{5}$, \\ SUNG-KEUM SEO ${ }^{5}$, IN-CHUL PARK ${ }^{5}$, CHONG SOON KIM ${ }^{5}$, YOUNG-WOO JIN ${ }^{4}$ and SUNG K. AHN ${ }^{1}$ \\ ${ }^{1}$ Functional Genoproteome Research Centre, Konkuk University, Seoul 143-701; ${ }^{2}$ Department of Obstetrics \\ and Gynecology, Konkuk University Hospital, Seoul 143-729; ${ }^{3}$ Department of Chemistry, Hanyang University, \\ Seoul 133-791; ${ }^{4}$ Division of Radiation Effect Research, Radiation Health Research Institute of KHNP, Seoul 132-703; \\ ${ }^{5}$ Laboratory of Functional Genomics, Korea Institute of Radiological and Medical Sciences, Seoul 139-706, Korea
}

Received November 12, 2007; Accepted February 22, 2008

\begin{abstract}
A-kinase-anchoring protein 149 (AKAP149) is a member of a structurally diverse, though functionally similar anchoring protein family and is localized to the outer membrane of mitochondria and in the endoplasmic reticulumnuclear envelope network. AKAP149 plays an important role in controlling the subcellular localization and temporal specificity of protein phosphorylation and mRNA metabolism by tethering kinases and phosphatases, such as protein kinase $\mathrm{A}$ and type I protein phosphatase, through its $\mathrm{N}$-terminal protein-binding motifs and mRNAs via its C-terminal RNAbinding motifs. It is well recognized that caspases play a central role in transducing and amplifying the intracellular death signal and that apoptosis is executed as a consequence of caspase-mediated cleavage of multiple cellular substrates. The identification of novel death substrates and elucidation of the consequences of their proteolytic cleavages by caspases are therefore crucial for our understanding of cell death and other biological processes. Herein, we demonstrated that AKAP149 is a direct substrate of active caspase-3, -8 -and -10 in vitro and in vivo. ${ }^{35}$ S-labeled full-length AKAP149 was completely cleaved in vitro by active caspase- $3,-8$ and -10 into two fragments of $\sim 105$ and $45 \mathrm{kDa}$, while caspase- 2 cleaved it partially and caspase-1 did not cleave it at all. AKAP149 was
\end{abstract}

Correspondence to: Dr Young-Woo Jin, Division of Radiation Effect Research, Radiation Health Research Institute of KHNP, Seoul 132-703, Korea

Dr Sung K. Ahn, Functional Genoproteome Research Centre, Konkuk University, 1 Hwayang-dong, Gwangjin-gu, Seoul 143-701, Korea

E-mail: ansfgrc@konkuk.ac.kr

*Contributed equally

Key words: A-kinase-anchoring protein 149, apoptosis, caspase, cleavage substrates also cleaved by caspases during Fas- and staurosporineinduced apoptosis in Jurkat $\mathrm{T}$ and HeLa cells, which were blocked by specific inhibitors of caspase- 3 and -8 . The specific cleavage site for these caspases was mapped in vitro and in vivo to Asp582 at AKAP149, which is located between the protein kinase A regulatory subunit anchoring and $\mathrm{KH}$ RNA-binding domains. In addition, HeLa cells transiently overexpressing AKAP149 D582E mutant were resistant to staurosporine-induced HeLa cell apoptosis. Taken together, these data suggest that AKAP149 activity may be deregulated by caspase-dependent proteolysis during apoptotic cell death and may provide useful information for elucidating the apoptosis signaling pathways in detail.

\section{Introduction}

cAMP activates protein kinase A (PKA), a broad specificity tetrameric Ser/Thr protein kinase and plays an essential role in the regulation of many important cellular activities $(1,2)$. PKA is comprised of two regulatory and two catalytic subunits. The binding of two cAMP molecules to each regulatory subunit promotes dissociation and activation of the catalytic subunit. As a consequence of this interaction, the catalytic subunit is able to catalyze the phosphorylation of local substrates. Specificity of PKA phosphorylation is achieved via the anchoring of PKA through the interaction of the regulatory subunit of PKA with A-kinase-anchoring proteins (AKAPs) (3-5). AKAPs are a family of $>50$ structurally diverse, though functionally similar anchoring proteins that have in common the ability to bind to the regulatory subunit of PKA. The targeting of AKAPs through this mechanism ensures that PKA is exposed to cAMP gradients locally generated by adenylyl cyclases and phosphodiesterases (PDEs) thus allowing for an appropriate substrate selection $(3,6)$.

AKAP149 (7), also designated AKAP1, is a human $149 \mathrm{kDa}$ anchoring protein homologous to mouse AKAP121 identified in the mitochondria (8-10) and in the endoplasmic reticulumnuclear envelope network (11). The anchoring of PKA by AKAP149 to mitochondria supports cAMP signaling and 
suppresses apoptosis $(12,13)$. PKA localization at this organelle promotes the phosphorylation and subsequent inactivation of the pro-apoptotic protein Bad (13). In addition, AKAP149 has been reported to tether to the protein phosphatase type I (PP1) regulatory subunit, which enhances PP1 phosphatase activity toward B-type lamins upon nuclear envelope reformation at mitosis exit, in order to promote lamin dephosphorylation and polymerization (11). AKAP149 also carry a K homology (KH) and a Tudor domain (10). These domains participate in various $\mathrm{RNA}$ /protein-binding interactions $(14,15)$, including associations with structural elements in the 3'-untranslated region (3'-UTR) of mRNAs and are responsible for regulating the localization, stabilization and translational regulation of these mRNAs (16-21).

It is now well recognized that apoptosis is executed by the caspase-mediated cleavage of multiple cellular substrates and that these substrates also act as transducers and amplifiers that determine the apoptotic threshold and cell fate (22-24). To date, 14 human and murine caspases have been identified and characterized $(23,24)$. Previously published studies have determined that caspase-1, $-4,-5$ and -11 are involved in cytokine maturation and inflammatory responses, while the remaining family members are primarily involved in apoptotic signaling pathways. Initiator caspases (caspase-2, -8, -9, -10 and -12) contain a long N-terminal prodomain which is essential for the recruitment of activation factors, while effector caspases (caspase-3, -6, -7 and -14) have a shorter prodomain, which requires cleavage by activated initiator caspases (22-24). As a consequence of the activity of effector caspases, or through the direct action of initiator caspases, the activity of many structural and regulatory proteins is altered; some are inactivated while other substrates can be activated. In addition to determining the apoptotic threshold and cell fate, caspase substrates can regulate the key morphological changes in apoptosis. Therefore, elucidating the consequences of caspasemediated proteolytic cleavage is critical for our understanding of cell death and other biological processes.

As a result of a systematic and genome-wide screen, we have recently identified five novel putative substrates, including murine AKAP129, of initiator caspase-8 and -10 in vitro (25). In our study, we present evidence that AKAP149, a human protein homologous to mouse AKAP121, is a bona fide proteolytic target that is specifically cleaved by caspases during apoptosis in cells. In addition, we present evidence in the present study demonstrating that AKAP149 activity is deregulated by caspase-dependent proteolysis during apoptotic cell death. The results of this study further our understanding of the mechanisms by which initiator caspase- 8 and -10 regulate apoptosis and as a result these studies will contribute to the further characterization of the pathways required for the regulation of apoptosis.

\section{Materials and methods}

Cell cultures and drug treatment. The human lung carcinoma cell line A549, acute lymphoblastic T cell line Jurkat and cervical cancer cell line HeLa were all obtained from ATCC (USA) and cultured in F12K, MEM, or RPMI-1640 media (Wellgene, Korea) supplemented with $10 \%$ heat-inactivated fetal bovine serum (Wellgene), $10 \mathrm{U} / \mathrm{ml}$ of penicillin
(Wellgene) and $100 \mathrm{mg} / \mathrm{ml}$ of streptomycin (Wellgene) in a humidified incubator with $5 \%$ of $\mathrm{CO}_{2}$ at $37^{\circ} \mathrm{C}$ atmosphere for the appropriate time. For inhibition experiments, Jurkat $\mathrm{T}$ and HeLa cells were pre-incubated with z-DEVD-fmk (Calbiochem, USA) or z-IETD-fmk (Calbiochem), cellpermeable and irreversible inhibitors of caspase-3 and -8 , respectively (26), for $30 \mathrm{~min}$ and subsequently treated with indicated amounts of apoptotic stimuli, the anti-Fas monoclonal antibody $\mathrm{CH}-11$ (Upstate, USA) and staurosporine (Sigma, USA) for $24 \mathrm{~h}$. The transfection of HeLa cells was performed by using the lipofectamine transfection reagent (Invitrogen, USA) according to the manufacturer's instructions.

Cloning of wild-type and D582E mutant of AKAP149. The entire open reading frame (ORF) of AKAP149 was cloned from cDNA of A549 cells into BamHI (New England Biolabs, USA) and EcoRI (New England Biolabs) sites of pCITE4a (Novagen, USA) with the following oligonucleotide primers (Bionics, Korea): sense, 5'-CGCGGATCCGCGATGGCAA TCCAGTTCCGT-3'; and anti-sense, 5'-GGAATTCCGGAA GGCTTGTGTAGTAGCT-3'. The ORF of AKAP149 was also subcloned into BamHI and EcoRI sites of pcDNA3.1-myc/ his A (Invitrogen) for expression in mammalian cells. Wildtype of AKAP149 inserted into pCITE4a and pcDNA3.1-myc/ his A plasmids was used as templates for creating the D582E mutant AKAP149: the Asp residue at position 582 of AKAP149 were mutated to Glu, using the QuickChange sitedirected mutagenesis kit (Stratagene, USA) according to the manufacturer's instructions with the following oligonucleotide primers (Bionics): 5'-GAACAGCATGGATTCCGTCCGTG GAGAGCTGTTGCAGTCTCAAGAAG-3' and 5'-CTTCTT GAGACTGCAACAGCTCTCCACGGAATCCATGCT GTTC-3'. Each construct was verified by automated DNA sequencing (Bionics) of the two strands.

Preparation of ${ }^{35}$ S-labeled proteins and proteolytic cleavage assay in vitro. ${ }^{35} \mathrm{~S}$-labeled AKAP149 proteins (wild-type and D582E) were transcribed and translated in vitro from pCITE4a-AKAP149 wild-type and D542E plasmids using the TnT coupled reticulocyte lysate system (Promega, USA) in the presence of ${ }^{35} \mathrm{~S}$-methionine (PerkinElmer, USA) according to the manufacturer's instructions. The labeled proteins were incubated with 10 units of recombinant and active human caspase- $1,-2,-3,-8$, or -10 (Calbiochem) in the caspase reaction buffer (20 mM Tris-HCl, pH 7.5, $10 \mathrm{mM}$ DTT and $0.1 \mathrm{mM}$ EDTA) at $37^{\circ} \mathrm{C}$ for $1 \mathrm{~h}$. Cleavage reactions were stopped by the addition of $4 \mathrm{x}$ SDS-sample buffer and boiled for $5 \mathrm{~min}$. Proteins were then separated by SDS-PAGE gel, dried using a gel-dryer with vacuum at $80^{\circ} \mathrm{C}$ for $1 \mathrm{~h}$ and were visualized by autoradiography.

Immunoblot analysis. Cells were collected, washed with cold PBS and lysed in lysis buffer (50 mM Tris- $\mathrm{HCl}, \mathrm{pH} 7.5$, $150 \mathrm{mM} \mathrm{NaCl}, 1 \%$ Nonidet P-40, $0.5 \%$ sodium deoxycholate and $0.1 \%$ SDS) supplemented with protease inhibitor cocktails (Roche, Germany). The lysates were clarified by centrifugation at $15,000 \mathrm{xg}$ for $15 \mathrm{~min}$ at $4^{\circ} \mathrm{C}$. Protein content was measured through the Bradford (Bio-Rad, USA) method. An aliquot (30 or $50 \mathrm{mg}$ of protein per lane) was resolved by SDS-PAGE and blotted to the nitrocellulose membrane. Immunoblotting 
was then carried out using the primary antibodies. Mouse anti-AKAP149 monoclonal antibody was purchased from BD Biosciences (USA), mouse anti- $\beta$-actin monoclonal antibody was from Sigma and rabbit anti-myc polyclonal antibodies were from Cell Signaling Technology (USA). The specific protein bands were visualized by SuperSignal West Pico Luminol (Pierce, USA) and exposed on Hyperfilm (GE Healthcare, USA).

Evaluation of apoptosis. Apoptosis was evaluated by staining cells with annexin V-FITC (BD Biosciences) and propidium iodide (PI, BD Biosciences). To quantitate apoptosis, prepared cells were washed with cold PBS and re-suspended in a binding buffer (10 mM Hepes/NaOH, pH 7.4, $140 \mathrm{mM}$ $\mathrm{NaCl}$ and $2.5 \mathrm{mM} \mathrm{CaCl}_{2}$ ) at a concentration of $1 \times 10^{6}$ cells $/ \mathrm{ml}$. Then, $5 \mathrm{ml}$ of annexin V-FITC and $10 \mathrm{ml}$ of PI were added to these cells and samples were analyzed with a FACScan flow cytometer (Calibur, BD Biosciences).

\section{Results and Discussion}

AKAP149 is proteolytically cleaved in vitro by active caspase-3, -8 and -10 . We recently reported (25) the construction of a mouse cDNA library and its use in performing a systematic and genome-wide screen for novel in vitro substrates of initiator caspases. As a consequence of these studies, we successfully identified five positive mouse cDNA fragments, including AKAP129, as putative caspase substrates (25). In our study, we examined whether AKAP149, a human homologous protein to mouse AKAP121, is a bona fide proteolytic target that is specifically cleaved by caspase(s) during in vivo apoptosis.

We examined whether full-length AKAP149 was cleaved by caspases in vitro. ${ }^{35} \mathrm{~S}$-labeled, full-length AKAP149 protein was transcribed and translated in vitro from pCITE4aAKAP149 plasmid using the TnT coupled reticulocyte lysate system was incubated with 10 units of recombinant and active human caspase- $1,-2,-3,-8$ or -10 . It was determined (Fig. 1) that in the presence of caspase- $3,-8$ or $-10,{ }^{35}$ S-labeled AKAP149 was completely cleaved into two fragments of $\sim 105$ and $45 \mathrm{kDa}$ while in the presence of caspase-2 or -1 , respectively, AKAP149 was either partially cleaved or remained intact. These results indicate that AKAP149 is a cleavage substrate of caspase-3, an effector caspase, as well as initiator caspase- 8 and -10 . In addition, these data demonstrate that AKAP149 is not a primary substrate of another initiator caspase, caspase-2. Furthermore, since caspase-1 has been implicated in cytokine maturation and inflammatory responses rather than in apoptotic cell death pathways $(23,24)$, the observations that caspase-1 does not elicit cleavage of AKAP149, while a number of pro-apoptotic caspases are able to do so, suggests that AKAP149 cleavage may occur predominantly during apoptotic cell death.

AKAP149 is cleaved by caspases in vivo during Fas-and staurosporine-induced apoptosis. Previous studies have thoroughly established that the induction of Fas receptor multimerization by their ligands or the anti-Fas monoclonal antibody $\mathrm{CH}-11$ results in the formation of the death inducing signaling complex (DISC) as well as the recruitment of

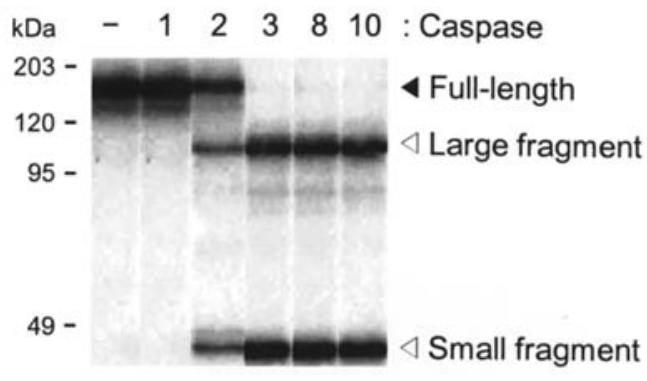

Figure 1. AKAP149 is proteolytically cleaved in vitro by active caspase-3, -8 and -10 . Ten microliters of the ${ }^{35}$ S-labeled full-length protein of AKAP149 was transcribed and translated in vitro from the pCITE4a-AKAP149 plasmid using the TnT coupled reticulocyte lysate system and incubated with 10 units of recombinant and active human caspase-1, $-2,-3,-8$ or -10 for $1 \mathrm{~h}$ at $37^{\circ} \mathrm{C}$. The cleavage products were separated by SDS-PAGE and detected by autoradiography. This experiment was performed in triplicate and the results of a representative experiment are shown. Black arrowhead, intact and fulllength form of AKAP149; white arrowhead, cleaved forms of AKAP149.

initiator caspase- 8 and $-10(23,24,27-29)$. The recruitment and oligomerization of caspase- 8 and -10 to DISC results in autocatalytic activation and is required for the initiation of cell death signaling pathways. In addition, it has been reported that AKAP149 is able to form a molecular complex with multiple protein kinases and phosphatases that play a critical role in the integration of signaling networks in cells; these proteins include PKA, PKC and PP1 (2,10-12,30,31).

Our observations (Fig. 1), lead us to examine whether AKAP149 was a bona fide cleavage target of caspases during cellular apoptotic death that was specifically triggered by cellular death receptors and/or the inhibition of protein kinase activity. Jurkat $\mathrm{T}$ and HeLa cells were treated for $24 \mathrm{~h}$ with an anti-Fas monoclonal antibody CH-11 $(25,50$ or $75 \mathrm{ng} / \mathrm{ml})$ and staurosporine, a cell-permeable and broad-spectrum inhibitor of protein kinases $(250,500$ or $750 \mathrm{nM})$, respectively. Cells were then analyzed for cleavage of AKAP149 by immunoblotting analysis while apoptotic cell death was evaluated by FACScan flow cytometry analysis. $\mathrm{CH}-11$ and staurosporine treatment of Jurkat $\mathrm{T}$ and HeLa cells resulted in a steady decrease of detectable levels of fulllength AKAP149 (Fig. 2A) and an increase of the cleavage product (data not shown). In addition, it was determined that the detection of decreased levels of AKAP149 was dependent on the dose of $\mathrm{CH}-11$ or staurosporine and the observed changes in full-length AKAP149 correlated with the apoptotic rates in these cells (Fig. 2A). Irradiation ( 0.5 to $10 \mathrm{~Gy}$ ) also resulted in the cleavage of AKAP149 in a dosedependent manner in the Jurkat $\mathrm{T}$ and HeLa cells (data not shown).

To confirm that $\mathrm{CH}-11$ - or staurosporine-dependent AKAP149 cleavage and apoptosis were directly affected by caspase-3, -8 and/or -10 in these cells, we assessed the effect of z-DEVD-fmk and z-IETD-fmk, cell-permeable and irreversible inhibitors of caspase-3 and -8 , respectively (26), using the same experimental sets. It was determined that following $24 \mathrm{~h}$ of culture, $40 \mu \mathrm{M}$ of either z-DEVD-fmk or z-IETD-fmk dramatically inhibited CH-11 (Fig. 2B) or staurosporine (Fig. 2C)-induced the cleavage of AKAP149 and apoptosis in these cells. Collectively, the data presented 
A

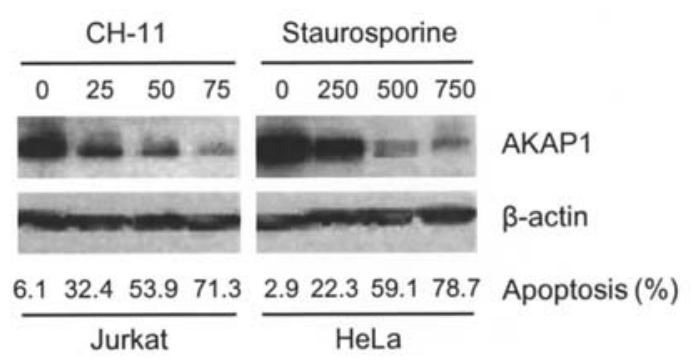

B

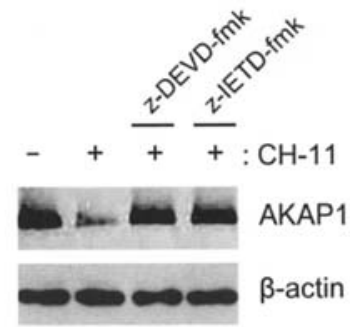

2.447 .48 .710 .0 Apoptosis (\%)

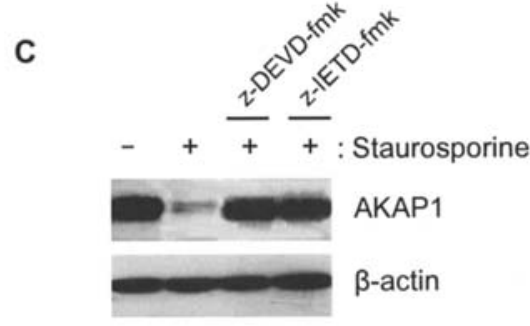

$\begin{array}{llll}4.0 & 62.1 & 9.9 & 13.5 \\ \text { Apoptosis (\%) }\end{array}$

Figure 2. AKAP149 is cleaved by caspases in vivo during Fas- and staurosporine-induced apoptosis in Jurkat T and HeLa cells. (A) Full-length AKAP149 protein levels are decreased in a dose-dependent manner during Fas- and staurosporine-induced Jurkat $\mathrm{T}$ and HeLa cell apoptosis. Jurkat $\mathrm{T}$ and HeLa cells were treated for $24 \mathrm{~h}$ with an anti-Fas monoclonal antibody (CH-11; 25,50 or $75 \mathrm{ng} / \mathrm{ml}$ ) and staurosporine, a cell-permeable and broad-spectrum inhibitor of protein kinases $(250,500$ or $750 \mathrm{nM})$, respectively. Equivalent protein concentrations were analyzed for cleavage of AKAP149 by Western blotting analysis. (B) Fas-induced proteolysis of AKAP149 is potently inhibited by inhibitors of caspase- 3 and -8 in Jurkat $\mathrm{T}$ cells. Jurkat $\mathrm{T}$ cells were preincubated for $30 \mathrm{~min}$ with $40 \mu \mathrm{M}$ of z-DEVD-fmk or z-IETD-fmk, cell-permeable and irreversible inhibitors of caspase- 3 and -8 , respectively and then treated for an additional $24 \mathrm{~h}$ with $50 \mathrm{ng} / \mathrm{ml}$ of $\mathrm{CH}-11$. (C) Staurosporineinduced proteolysis of AKAP149 is potently inhibited by inhibitors of caspase-3 and -8 in HeLa cells. HeLa cells were preincubated for 30 min with $40 \mu \mathrm{M}$ of z-DEVD-fmk or z-IETD-fmk and then treated for an additional $24 \mathrm{~h}$ with $500 \mathrm{nM}$ of staurosporine. B-actin expression levels were included as a loading control. For each condition, a percentage of apoptosis of the corresponding cell population was determined by FACScan flow cytometry analysis. This experiment was performed in triplicate and the results of a representative experiment are shown.

in Figs. 1 and 2 indicate that AKAP149 is proteolytically cleaved in cells during apoptosis, as cleavage is dependent upon the activity of caspase- $-3,-8$ and -10 .

It has been reported that the anchoring of PKA by AKAP149 to mitochondria supports cAMP signaling (12). PKA localization at the mitochondrial region promotes phosphorylation and inactivation of the pro-apoptotic protein Bad (13). Additionally, evidence demonstrates that AKAP149 also tethers PP1 resulting in enhanced PP1 phosphatase activity and promoting lamin dephosphorylation and
A

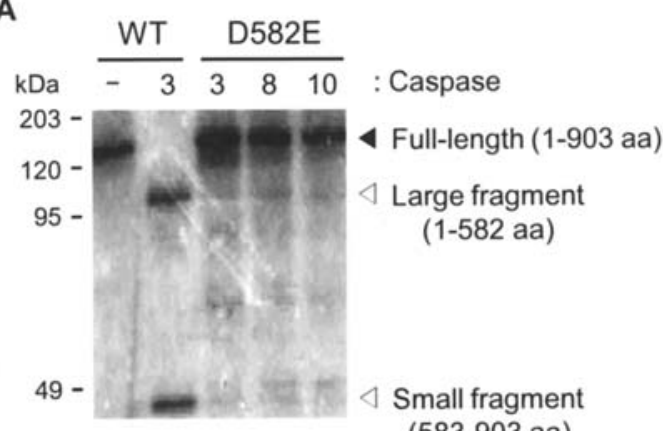

(583-903 aa)

B

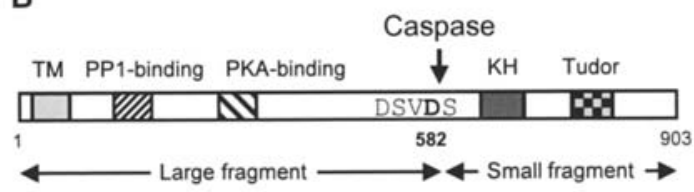

Figure 3. AKAP149 is cleaved in vitro by caspase-3, -8 and -10 at Asp582. (A) AKAP149 D582E mutant is resistant to cleavage by caspase-3, -8 and -10 in vitro. AKAP149 D582E mutant was created from the wild-type (WT) of pCITE4a-AKAP149 by site-directed mutagenesis as described in Materials and methods. Ten microliters of ${ }^{35} \mathrm{~S}$-labeled wild-type and D582E mutant (D582E) of AKAP149 were incubated with or without 10 units of recombinant and active human caspase- $3,-8$ or -10 for $1 \mathrm{~h}$ at $37^{\circ} \mathrm{C}$. The cleavage products were separated by SDS-PAGE and detected by autoradiography. This experiment was performed in triplicate and the results of a representative experiment are shown. Black left triangular arrow, intact and full-length form of AKAP1; white left triangular arrow, cleaved forms of AKAP1. (B) A schematical representation of structural domains and a caspase cleavage residue of AKAP149. The amino acid positions are indicated. TM, predicted transmembrane domain (6-28); PP1-binding, PP1-binding domain (153-157); PKA-binding, PKA regulatory subunitbinding domain (355-377); KH, KH domain (606-676); Tudor, Tudor domain (756-815) and aa, amino acids, are indicated.

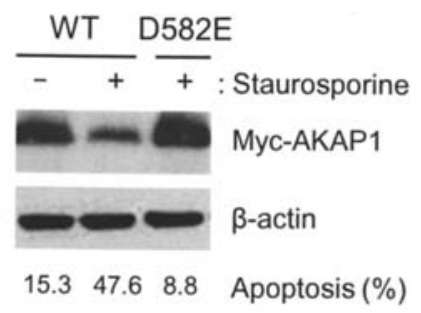

Figure 4. The Asp582 residue of AKAP149 is responsible for its proteolytic cleavage during staurosporine-induced HeLa cell apoptosis. HeLa cells were transiently transfected with either wild-type (WT) or D582E mutant (D582E) AKAP149 inserted into pcDNA3.1-myc/his plasmids for $24 \mathrm{~h}$, followed by treatment with $500 \mathrm{nM}$ of staurosporine for an additional $24 \mathrm{~h}$. After harvesting the cells, equal protein concentrations were prepared and subsequently analyzed for levels of exogenously expressed AKAP149 by immunoblotting analysis using rabbit anti-myc polyclonal antibodies. A mouse anti- $\beta$-actin monoclonal antibody was used as a control to verify that equivalent amounts of protein were loaded in each lane. For each condition, a percentage of apoptosis of the corresponding cell was determined by FACScan flow cytometry analysis. This experiment was performed in triplicate and the results of a representative experiment are shown.

polymerization (11). These activities are regulated by the phosphorylation of sites flanking the AKAP149 RVXF motif (30). Furthermore, it has been reported that binding of mRNAs to the mitochondrial AKAP121 is dependent on AKAP121 phosphorylation by PKA, which may facilitate the import of 
mitochondrial mRNAs via AKAP121 in response to cAMP stimulation and PKA activation (10). Therefore, it may be possible that, upon triggering apoptotic signals in Jurkat $\mathrm{T}$ cells through Fas receptor transduction, activated initiator caspases, caspase- 8 and -10 , begin to directly cleave AKAP149 during the early stages of apoptosis. AKAP149 cleavage is completed by downstream effector caspases of caspase- 8 and -10 following their complete activation. When protein kinase signaling pathways are blocked in cells, or levels of cellular cAMP are repressed, various intracellular apoptotic signals are triggered and result in the simultaneous activation of caspase- $3,-8$ and -10 and as a consequence may stimulate the direct cleavage of AKAP149. It remains to be determined whether AKAP149 cleavage occurs prior to Bid cleavage by initiator caspases or mitochondrial damage (27-29).

AKAP149 is cleaved by caspases at Asp582 in vitro and in vivo. In an effort to more completely characterize the caspase(s) responsible for AKAP149 proteolysis during apoptotic cell death, we then investigated the precise cleavage site of AKAP149 by caspase- $3,-8$ and -10 . Our previous studies of the in vitro and in vivo cleavage patterns of AKAP149 (Figs. 1 and 2 and data not shown) suggested that caspase-3, -8 and -10 were likely to share a single cleavage site that may localize to a residue of approximately one third from either terminus of the full-length AKAP149 protein. From these, we narrowed down to a plausible residue at Asp-Ser-Val-Asp582 (DSVD582) as a potential cleavage site of AKAP149 by caspases. To confirm that it is indeed the cleavage site for caspase-3, -8 and -10, we mutated the Asp582 residue to Glu (D582E) by site-directed mutagenesis. It was determined (Fig. 3) that the substitution of Asp582 with Glu completely prevented cleavage by caspas-3, -8 and -10 and the 105 and $45 \mathrm{kDa}$ cleavage fragments were not formed in vitro.

These observations were confirmed in vivo. HeLa cells were transiently transfected with either wild-type or the D582E mutant of AKAP149 for $24 \mathrm{~h}$, followed by treatment with $500 \mathrm{nM}$ of staurosporine for an additional $24 \mathrm{~h}$. Equal protein concentrations were then analyzed for levels of exogenously expressed AKAP149 by immunoblot analysis and for the percentage of apoptosis was determined for the corresponding cell populations by FACScan flow cytometry analysis. As shown in Fig. 4, levels of exogenously expressed D582E mutant, though not wild-type AKAP149 protein, were not changed in HeLa cells treated with $500 \mathrm{nM}$ of staurosporine for $24 \mathrm{~h}$. In addition, we concluded that cells transiently overexpressing the AKAP149 D582E mutant were also resistant to staurosporine-induced apoptosis.

The identified structural domains of AKAP149, which are schematically outlined in Fig. 3B, are highly conserved when compared to the structural domains of AKAP121 $(4,7,8,10-12,30,32)$. Consistent with AKAP121, the apoptotic cleavage site of AKAP149 lies between the PKA regulatory subunit anchoring and KH RNA-binding domains. In addition, AKAP149 contains an N-terminal transmembrane domain (7) and harbors a putative consensus binding site for PP1 $(11,30,32)$. Previous studies have reported that AKAP149 recruits the PP1 regulatory subunit to the nuclear envelope upon somatic nuclear reformation and that the sequestering of PP1 to the nuclear envelope is a prerequisite for assembly of
B-type lamins (11). Additionally, the anchoring of PKA by AKAP149 to the mitochondria has been reported to support cAMP signaling and suppresses apoptosis by promoting the phosphorylation and subsequent inactivation of the proapoptotic protein $\operatorname{Bad}(12,13)$.

Additional studies here have determined that the C-terminal small cleavage fragment (583-903 amino acids) of AKAP149, which is produced as a consequence of caspase-mediated cleavage of full-length AKAP149 during apoptosis possesses a KH and a Tudor domain. The KH RNA binding domain has been reported to be essential for mediating the binding of AKAP121 to mRNA $(35,36)$ and mRNA binding is positively regulated by phosphorylation of the $\mathrm{KH}$ domain by PKA and cAMP. Consistent with this reported role of the AKAP121 $\mathrm{KH}$ domain, it has been demonstrated that AKAP121 binds to the 3'-UTR of mRNAs that encode Fo-f, a subunit of mitochondrial ATP synthase and manganese superoxide dismutase, a mitochondrial protein involved in the reduction of oxidative stress (10). Furthermore, AKAP121 has been shown to bind to the mRNA 3'-UTR of lipoprotein lipase (LPL), an extracellular enzyme hydrolyzing the triglyceride core of circulating chylomicrons $(33,34)$. This interaction is critical for the decrease in the extent of LPL mRNA translation following PKA stimulation in white adipose tissue. In addition to a KH domain, AKAP149 contains a Tudor domain, which is also found in many RNA-interacting proteins (37). Tudor domains may also be involved in DNA binding (38) and protein-protein interaction through binding to Arg-Gly (RG) motifs $(39,40)$ and to methylated lysine in core histone H3 $(41,42)$.

The data presented in this study, support the hypothesis that during apoptotic cell death, AKAP149 is cleaved specifically at residue Asp582 by caspase- $3,-8$ and -10 resulting in the complete loss of the $\mathrm{C}$-terminal $\mathrm{KH}$ and Tudor domains. Following cleavage, our data suggest that the $\mathrm{N}$ terminal large fragment (1-582 amino acids) may still be able to tether protein kinases and phosphatases to the mitochondrial and nuclear membranes thus enabling these molecules to maintain their activity. We cannot, however, exclude the possibility that the N-terminal cleavage product of AKAP149 may somehow lose some, if not all, biological functions. Additional studies are required to fully examine this possibility.

Collectively our data demonstrate that AKAP149 is a novel death substrate that is directly targeted by active caspase- $3,-8$ and -10 in vitro and in vivo. In addition, the results of these studies suggest that AKAP149 activity may be deregulated by caspase-dependent proteolysis during apoptotic cell death. While additional studies will be required to fully elucidate the apoptosis signaling pathways, these data extend our understanding of the signaling mechanisms and key components required to regulate this process.

\section{Acknowledgements}

We are grateful to RHRI of KHNP and all other members in our research group for their support and advice in regards to this report. The study reported here was supported by Konkuk University in 2004. 


\section{References}

1. Tasken K and Aandahl EM: Localized effects of cAMP mediated by distinct routes of protein kinase A. Physiol Rev 84: 137-167, 2004.

2. Taylor SS, Kim C, Vigil D, Haste NM, Yang J, Wu J and Anand GS: Dynamics of signaling by PKA. Biochim Biophys Acta 1754: 25-37, 2005

3. Michel JJ and Scott JD: AKAP mediated signal transduction. Annu Rev Pharmacol Toxicol 42: 235-257, 2002.

4. Carlson CR, Ruppelt A and Tasken K: A kinase anchoring protein (AKA) interaction and dimerization of the RI $\alpha$ and RIß, regulatory subunits of protein kinase A in vivo by the yeast two hybrid system. J Mol Biol 327: 609-618, 2003.

5. Wong W and Scott JD: AKAP signalling complexes: focal points in space and time. Nat Rev Mol Cell Biol 5: 959-970, 2004.

6. McConnachie G, Langeberg LK and Scott JD: AKAP signaling complexes: getting to the heart of the matter. Trends Mol Med 12: 317-323, 2006.

7. Trendelenburg G, Hummel M, Riecken EO and Hanski C: Molecular characterization of AKAP149, a novel A kinase anchor protein with a $\mathrm{KH}$ domain. Biochem Biophys Res Commun 225: 313-319, 1996

8. Chen Q, Lin RY and Rubin CS: Organelle-specific targeting of protein kinase AII (PKAII). Molecular and in situ characterization of murine A kinase anchor proteins that recruit regulatory subunits of PKAII to the cytoplasmic surface of mitochondria. J Biol Chem 272: 15247-15257, 1996.

9. Feliciello A, Rubin CS, Avvedimento EV and Gottesman ME: Expression of a kinase anchor protein 121 is regulated by hormones in thyroid and testicular germ cells. J Biol Chem 273: 23361-23366, 1998.

10. Ginsberg MD, Feliciello A, Jones JK, Avvedimento EV and Gottesman ME: PKA-dependent binding of mRNA to the mitochondrial AKAP121 protein. J Mol Biol 327: 885-897, 2003.

11. Steen RL, Martins SB, Tasken K and Collas P: Recruitment of protein phosphatase 1 to the nuclear envelope by A-Kinase anchoring protein AKAP149 is a prerequisite for nuclear lamina assembly. J Cell Biol 150: 1251-1262, 2000.

12. Affaitati A, Cardone L, de Cristofaro T, Carlucci A, Ginsberg MD, Varrone S, Gottesman ME, Avvedimento EV and Feliciello A: Essential role of A-kinase anchor protein 121 for cAMP signaling to mitochondria. J Biol Chem 278: 4286-4294, 2003.

13. Harada H, Becknell B, Wilm M, Mann M, Huang LJ, Taylor SS, Scott JD and Korsmeyer SJ: Phosphorylation and inactivation of BAD by mitochondria-anchored protein kinase A. Mol Cell 3: 413-422, 1999.

14. Burd CG and Dreyfuss G: Conserved structures and diversity of functions of RNA-binding proteins. Science 265: 615-621, 1994.

15. Siomi H, Matunis MJ, Michael WM and Dreyfuss G: The premRNA binding $\mathrm{K}$ protein contains a novel evolutionarily conserved motif. Nucleic Acids Res 21: 1193-1198, 1993.

16. Havin L, Git A, Elisha Z, Oberman F, Yaniv K, Schwartz SP, Standart N and Yisraeli JK: RNA-binding protein conserved in both microtubule- and microfilament-based RNA localization. Genes Dev 12: 1593-1598, 1998.

17. Ross AF, Oleynikov Y, Kislauskis EH, Taneja KL and Singer RH: Characterization of a beta-actin mRNA zipcode-binding protein. Mol Cell Biol 17: 2158-2165, 1997.

18. Zehner ZE, Shepherd RK, Gabryszuk J, Fu TF, Al-Ali M and Holmes WM: RNA-protein interactions within the 3'-untranslated region of vimentin mRNA. Nucleic Acids Res 25: 3362-3370, 1997.

19. Bassell GJ, Oleynikov Y and Singer RH: The travels of mRNAs through all cells large and small. FASEB J 13: 447-454, 1999.

20. Hesketh JE: mRNA targeting: signals in the 3'-untranslated sequences for sorting of some mRNAs. Biochem Soc Trans 24: 521-527, 1996.

21. Oleynikov Y and Singer RH: RNA localization: different zipcodes, same postman? Trends Cell Biol 8: 381-383, 1998.
22. Fischer U, Janicke RU and Schulze-Osthoff K: Many cuts to ruin: a comprehensive update of caspase substrates. Cell Death Differ 10: 76-100, 2003.

23. Degterev A, Boyce M and Yuan J: A decade of caspases. Oncogene 22: 8543-8567, 2003.

24. Philchenkov A: Caspases: potential targets for regulating cell death. J Cell Mol Med 8: 432-444, 2004.

25. Bae S, Ha T-S, Yoon Y, Lee J, Cha HJ, Yoo H, Choe T-B, Li S, Sohn I, Kim J-Y, Kim C-S, Jin H-O, Lee H-C, Park I-C, Kim CS, Jin Y-W and Ahn SK: Genome-wide screening and identification of novel proteolytic cleavage targets of caspase- 8 and -10 in vitro. Int J Mol Med 21: 381-386, 2008.

26. Thornberry NA and Lazebnik Y: Caspases: enemies within. Science 281: 1312-1316, 1998

27. Li H, Zhu H, Xu CJ and Yuan J: Cleavage of BID by caspase 8 mediates the mitochondrial damage in the Fas pathway of apoptosis. Cell 94: 491-501, 1998.

28. Milhas D, Cuvillier O, Therville N, Clave P, Thomsen M, Levade T, Benoist $\mathrm{H}$ and Segui B: Caspase-10 triggers Bid cleavage and caspase cascade activation in FasL-induced apoptosis. J Biol Chem 280: 19836-19842, 2005.

29. Kischkel FC, Lawrence DA, Tinel A, LeBlanc H, Virmani A, Schow P, Gazdar A, Blenis J, Arnott D and Ashkenazi A: Death receptor recruitment of endogenous caspase-10 and apoptosis initiation in the absence of caspase-8. J Biol Chem 276: 46639-46649, 2001

30. Küntziger T, Rogne M, Folstad RL and Collas P: Association of PP1 with its regulatory subunit AKAP149 is regulated by serine phosphorylation flanking the RVXF motif of AKAP149. Biochemistry 45: 5868-5877, 2006.

31. Taylor SS, Yang J, Wu J, Haste NM, Radzio-Andzelm E and Anand GS: PKA: a portrait of protein kinase dynamics. Biochim Biophys Acta 1697: 259-269, 2004.

32. Zhao S and Lee EY: A protein phosphatase-1-binding motif identified by the panning of a random peptide display library. J Biol Chem 272: 28368-28372, 1997.

33. Ranganathan G, Phan D, Pokrovskaya ID, McEwen JE, Li C and Kern PA: The translational regulation of lipoprotein lipase by epinephrine involves an RNA binding complex including the catalytic subunit of protein kinase A. J Biol Chem 277: 43281-43287, 2002.

34. Ranganathan G, Pokrovskaya I, Ranganathan S and Kern PA: Role of A kinase anchor proteins in the tissue-specific regulation of lipoprotein lipase. Mol Endocrinol 19: 2527-2534, 2005.

35. Siomi $\mathrm{H}$ and Dreyfuss G: RNA-binding proteins as regulators of gene expression. Curr Opin Genet Dev 7: 345-353, 1997.

36. Adinolfi S, Bagni C, Castiglione Morelli MA, Fraternali F, Musco G and Pastore A: Novel RNA-binding motif: The KH module. Biopolymers 51: 153-164, 1999.

37. Ponting CP: Tudor domains in proteins that interact with RNA. Trends Biochem Sci 22: 51-52, 1997.

38. Charier G, Couprie J, pha-Bazin B, Meyer V, Quemeneur E, Guerois R, Callebaut I, Gilquin B and Zinn-Justin S: The Tudor tandem of 53BP1: A new structural motif involved in DNA and RG-rich peptide binding. Structure 12: 1551-1562, 2004.

39. Meister G, Eggert C and Fischer U: SMN-mediated assembly of RNPs: A complex story. Trends Cell Biol 12: 472-478, 2002.

40. Selenko P, Sprangers R, Stier G, Buhler D, Fischer U and Sattler M: SMN tudor domain structure and its interaction with the Sm proteins. Nat Struct Biol 8: 27-31, 2001.

41. Huang Y, Fang J, Bedford MT, Zhang Y and Xu RM: Recognition of histone H3 lysine-4 methylation by the double tudor domain of JMJD2A. Science 312: 748-751, 2006.

42. Huyen Y, Zgheib O, Ditullio RA Jr, Gorgoulis VG, Zacharatos P, Petty TJ, Sheston EA, Mellert HS, Stavridi ES and Halazonetis TD: Methylated lysine 79 of histone H3 targets 53BP1 to DNA double-strand breaks. Nature 432: 406-411, 2004. 